\title{
Función cognitiva y composición corporal en mujeres adultas mayores*
}

\author{
Leonardo Rodríguez Perdomo** \\ Jaime Sneider Barón Cuervo***
}

Recibido: junio 05 de 2019 • Aceptado: agosto 16 de 2019

\section{Resumen}

La prueba de evaluación cognitiva de Montreal (MoCA, por sus siglas en inglés) es un instrumento para medir el deterioro cognitivo leve y estados de demencia en adultos mayores, y presenta una correlación frente al componente morfológico. Se estudiaron 54 sujetos de sexo femenino, entre los 60-70 años y se registraron variables morfológicas con el equipo de impedancia SECA mBCA514 ${ }^{\circledR}$ : masa magra, masa grasa, masa muscular y masa muscular segmentada en cinco compartimentos; las funciones cognitivas se evaluaron con el test MoCA. Los resultados se reportaron en medias y desviación estándar en el componente morfológico: edad (años [63.9 \pm 3.3$]$ ), talla (metros $[\mathrm{m}][1.53 \pm 0.07]$ ), peso (kilogramos $[\mathrm{kg}][67.04 \pm 12.2]$ ), perímetro de la cintura (centímetros $[\mathrm{cm}][94.4 \pm 11.6]$ ), índice de masa corporal (IMC) $(27.6 \pm 3.7)$, masa grasa $(\mathrm{kg}[26.4 \pm 7.4])$, masa magra $(\mathrm{kg}$ $[40.6 \pm 7.3])$ y masa muscular segmentada en cinco compartimentos. Para

"Artículo de Investigación, con financiación del Centro de formación en Actividad física y cultura - SENA. Citar como: Rodríguez, L. y Barón, J. (2019). Función cognitiva y composición corporal en mujeres adultas mayores. Revista de Investigación Cuerpo, Cultura y Movimiento, 9(1), 45-58. DoI: https://doi.org/10.15332/2422474x/5351

"* Magíster en Ciencias y Tecnologías del Deporte y la Actividad Física de la Universidad Manuela Beltrán, independiente, Bogotá, Colombia.

Correo electrónico: leonardpersonal@misena.edu.co / ORCID: 0000-0003-2743-7168.

*** Especialista en Nutrición para el Deporte y la Actividad Física de la Universidad de León, Funniber, independiente, Bogotá, Colombia. Correo electrónico: jsbaron86@misena.edu.co / ORCID: 0000-0002-9280-2128. 
la variable cognitiva con el MoCA: visoespacial $(2.4 \pm 1.1)$, denominación animal $(2.6 \pm 0.5)$, memoria $(1.6 \pm 1.8)$, atención $(3.3 \pm 2.0)$, lenguaje (1.5 $\pm 1.0)$, abstracción $(1.3 \pm 0.56)$, orientación $(5.7 \pm 0.52)$, total $(18.3 \pm 5.5)$.

Palabras clave: cognitiva, composición corporal, mujeres, adulto mayor. 


\section{Cognitive function and body composition in older adult women}

\section{Abstract}

The Montreal Cognitive Assessment (MoCA) Test is an instrument to measure mild cognitive impairment and dementia condition in older adults and has a correlation with the morphological component. Fifty-four (54) female individuals, between 60-70 years old, were studied and morphological variables were recorded with the SECA mBCA $514^{\circledR}$ impedance equipment: lean mass, fat mass, muscle mass and segmented muscle mass in five compartments. Cognitive functions were evaluated with the MoCA test. The results were reported in means and standard deviation in the morphological component: age (years [63.9 \pm 3.3$]$ ), height (meters [m] [1.53 \pm 0.07$]$ ), weight (kilograms $[\mathrm{kg}][67.04 \pm 12.2]$ ), waist circumference (centimeters $[\mathrm{cm}][94.4 \pm 11.6]$ ), body mass index (BMI) $(27.6 \pm 3.7)$, fat mass $(\mathrm{kg}[26.4 \pm 7.4])$, lean mass $(\mathrm{kg}$ $[40.6 \pm 7.3])$ and segmented muscle mass in five compartments. For the cognitive variable with MoCA: visuospatial $(2.4 \pm 1.1)$, animal denomination $(2.6 \pm 0.5)$, memory $(1.6 \pm 1.8)$, attention $(3.3 \pm 2.0)$, language $(1.5 \pm 1.0)$, abstraction $(1.3 \pm 0.56)$, orientation $(5.7 \pm 0.52)$, total $(18.3 \pm 5.5)$.

Keywords: cognitive, body composition, women, elderly. 


\section{Introducción}

El envejecimiento es un proceso que afecta diferentes factores del ser humano (Tartt, et al., 2018). Dentro de las afectaciones se presentan cambios en los ámbitos funcionales, corporales, emocionales y cognitivos (Erickson, Hillman y Kramer, 2015). Hoy día, en Colombia existen más de 5.2 millones de adultos mayores, que es aproximadamente el $10.8 \%$ del total de la población. Se calcula que para el 2050 serán más de 14.1 millones de adultos mayores, un total del $23 \%$ de la población comprendida dentro del rango de edad mayor de 60 años (Montoya, et al., 2015).

El deterioro cognitivo leve (DCL) es conocido como una afectación en la que el sujeto da muestras de presentar pérdidas de tipo cognitivo en algunas acciones que están relacionadas con el lenguaje, la acción visoespacial y la memoria, según reporta la Academia Americana de Neurología (Petersen, et al., 2018). Se ha encontrado que el DCL es el primer paso para el desarrollo de la enfermedad de Alzheimer (Freitas, Simões, Alves, y Santana, 2013), así como para la generación de algunos trastornos de tipo neurodegenerativo y psiquiátrico (Huey, et al., 2013).

El DCL se presenta en el $18 \%$ de los sujetos entre los 55 y > 60 años (Hu, et al., 2017). Esta afectación presenta un aumento del $10 \%$ en edades comprendidas entre 70 y 75 años y un aumento del $25 \%$ en edades comprendidas entre los 80 y 85 años (Mitchell y Shiri-Feshki, 2009). La predominancia de daño cognitivo que se presenta en América Latina es de 6 a 7 casos en una muestra poblacional de 100 sujetos mayores de 60 años y se pronostica un aumento de $140 \%$ a 2040 (Parra, et al., 2018).

La American Academy of Neurology (AAN, 2018) recomienda el uso del test Montreal Cognitive Assessment (MoCA) como el primer paso para la detección del DCL (Petersen, et al., 2018). El MoCA es un test de cribado, desarrollado aproximadamente en el año 2005, con una duración de 10 minutos según las características de la población (Loureiro, Garcia, Adana, Yacelga, Rodriguez-Lorenzana y Maruta, 2018). Este test evalúa las capacidades funcionales ejecutivas, la capacidad visoespacial, la memoria, la concentración, la atención, el lenguaje y la orientación, y presenta una puntuación máxima perfecta de treinta puntos (Zhou, et al., 2014).

Aunque el test ha sido validado en diferentes idiomas y continentes (Delgado, Araneda, y Behrens, 2019), presenta algunas limitaciones frente al nivel académico y socioeconómico de los sujetos a quienes se les practica. 
Por lo tanto, se recomienda tener en cuenta el nivel económico, social y académico en el momento de la aplicación (Romo-Galindo y Padilla-Moya, 2019). Estudios de validación en Colombia sugieren que la prueba de MoCA presenta una pobre discriminación en poblaciones analfabetas o de bajo nivel de escolaridad, y presentan un punto de corte más bajo que el que muestra el autor principal (Gómez, Zunzunegui, Lord, Alvarado y García, 2013).

El papel de la actividad física frente al componente funcional y morfológico se ha evidenciado por muchos años. Ahora bien, el componente cognitivo y la actividad física -que se ve reflejada en el comportamiento funcional y morfológico- son asuntos que se han venido investigando con gran interés (Rodríguez-Romo, Barriopedro, Alonso y Garrido-Muñoz, 2015). En diferentes estudios se evidencia la relación existente entre la composición corporal y las acciones funcionales en adultos mayores (Gianoudis, Bailey y Daly, 2015). Este estudio busca determinar el nivel de correlación entre el comportamiento morfológico y el nivel cognitivo en una población de mujeres adultas entre 60 y 70 años en la ciudad de Bogotá.

\section{Metodología}

El presente es un estudio transversal descriptivo (cross-sectional transversal) orientado a determinar el nivel de correlación existente entre el test de MoCA para determinar el DCL y la composición corporal, en un grupo de 54 mujeres con edades contenidas entre 60 y 70 años (tabla 1).

Tabla 1. Cronograma metodológico de la investigación

\begin{tabular}{ll}
\hline & Cronograma metodológico \\
\hline Componente & $\begin{array}{l}\text { Peso, talla, IMC, \% grasa, \% muscular, masa mus- } \\
\text { cular segmentada (músculo tronco, músculo pierna } \\
\text { morfológico } \\
\text { derecha, músculo pierna izquierda, músculo brazo } \\
\text { derecho y músculo brazo izquierdo) } \\
\text { Test MoCA }\end{array} \quad \begin{array}{c}\text { Componentes: visoespacial, denominación, memoria, } \\
\text { lenguaje, abstracción, orientación }\end{array}$ \\
\hline Muestra: 54 mujeres de la ciudad de Bogotá
\end{tabular}

Fuente: elaboración propia. 
La muestra fue seleccionada por convocatoria abierta y los criterios de inclusión utilizados fueron mujeres físicamente activas durante los últimos 12 meses, con una intervención mínimo de tres veces por semana en un programa dirigido y controlado, sin ningún consumo de sustancias que afectaran las variables funcionales, como la prueba cognitiva. Se excluyeron sujetos que presentaran estudios inferiores a la primera fase educativa (primaria básica - 5 años). Durante la prueba fueron excluidas mujeres que presentaran algunos comportamientos asociados con el cansancio, sueño y desinterés.

El estudio cumple con lo establecido en la Declaración de Helsinki (2000). Todas las participantes fueron informadas sobre los procedimientos, características, riesgos del estudio y uso de la información, por medio de un consentimiento informado, el cual fue firmado por cada una.

Con el fin de buscar un mayor control de los resultados, tanto de composición corporal como el test de MoCA, todas las pruebas se tomaron en horas de la mañana, controlando la calidad del sueño o el consumo de algún medicamento. Por lo tanto, si se encontraba alguna de estas características que afectara la prueba, las participantes se citaban en una nueva jornada de evaluación pasando por el mismo procedimiento.

\section{Instrumentos de medición}

Para el registro de la talla se usó un tallímetro SECA 264 ${ }^{\circledR}$ (Hamburgo, Alemania). La composición corporal se tomó con un SECA mBCA514 ${ }^{\circledR}$ (Aristizábal y Giraldo, 2017) y para la prueba cognitiva se utilizó el test de MoCA (Sala, et al., 2019).

\section{Conflicto de intereses}

El resultado de la investigación hace parte de un proyecto realizado en el Servicio Nacional de Aprendizaje (SENA), por el grupo de investigación en Actividad Física y Deporte de Bogotá. No se presentó ningún conflicto de intereses por parte de los autores. 


\section{Resultados}

El análisis estadístico se realizó con el software spss, versión 21 (Chicago, IL, USA). Se presentaron pruebas de normalidad y descriptivos en medias y desviación estándar. Se determinó el coeficiente de correlación de Pearson (Leal, López, Martínez, Tapia y de León, 2019) entre las variables morfológicas y el test de MoCA. La tabla 2 presenta los resultados con una normalidad p > 0.05 y descriptivos del componente morfológico, en el que se resalta un IMC de $27.9 \pm 3.34$, el cual corresponde a resultados de sobrepeso en la población. Esto determina un primer riesgo de salud y afectaciones de tipo metabólico, según lo determinado por la Organización Mundial de las Salud (oms) (Labraña, et al., 2017). Se encuentra un perímetro de cintura de $92,3 \pm 9,16$, que diagnostica obesidad abdominal, la cual genera unos trastornos de tipo metabólico y riesgo cardiovascular (Buendía, Zambrano, Díaz, Reino, Ramírez y Espinoza, 2016). La obesidad abdominal también está asociada con la resistencia a la insulina, disminución del colesterol HDL, presencia de diabetes tipo II y riesgo aumentado de sufrir hipertensión arterial (Gaur y Ranga, 2018).

Tabla 2. Resultados descriptivos del componente morfológico

\begin{tabular}{lcccccccccc}
\hline & Edad & $\begin{array}{c}\text { Perímetro } \\
\text { de } \\
\text { cintura }\end{array}$ & $\begin{array}{c}\text { Peso } \\
(\mathrm{kg})\end{array}$ & $\begin{array}{c}\text { Talla } \\
(\mathrm{m})\end{array}$ & $\begin{array}{c}\text { IMC } \\
(\mathrm{kg} / \mathrm{m})\end{array}$ & $\begin{array}{c}\text { Masa } \\
\text { grasa } \\
(\mathrm{kg})\end{array}$ & $\begin{array}{c}\text { Masa } \\
\text { magra } \\
(\mathrm{kg})\end{array}$ & $\begin{array}{c}\text { Índice } \\
\text { de masa } \\
\text { grasa }\end{array}$ & $\begin{array}{c}\text { Masa } \\
\text { musculoes- } \\
\text { quelética } \\
(\mathrm{kg})\end{array}$ & $\begin{array}{c}\text { Grasa } \\
\text { visceral }\end{array}$ \\
\hline Media & 63.9 & 94.4 & 67.04 & 1.53 & 27.6 & 26.4 & 40,6 & 14.7 & 17.972 & 3.2 \\
Des. Est & \pm 3.3 & \pm 11.6 & \pm 12.2 & \pm 0.07 & \pm 3.7 & \pm 7.4 & \pm 7.3 & \pm 6.5 & \pm 3.51 & \pm 1.1 \\
Mínimo & 60 & 74 & 50.1 & 1.45 & 22.6 & 13.1 & 31 & 7 & 10.2 & 2 \\
Máximo & 70 & 113 & 90 & 1.73 & 34.9 & 39.5 & 55 & 39 & 30.2 & 6 \\
\hline
\end{tabular}

Fuente: elaboración propia.

En la tabla 3 se muestran los rangos de normalidad $\mathrm{p}>0.05$ y descriptivos del componente morfológico muscular segmentado en cinco compartimentos: músculo del tronco, músculo brazo derecho (BD), músculo brazo izquierdo (BI), músculo pierna derecha (PD) y músculo pierna izquierda (PI). 
Los resultados presentan una alta homogeneidad en todos los segmentos evaluados.

Tabla 3. Resultados descriptivos componente muscular segmentado

\begin{tabular}{lccccc}
\hline & Músculo tronco & Músculo вD & Músculo вI & Músculo PD & Músculo PI \\
\hline Media & 8.9 & 1.0 & 1.0 & 3.6 & 3.9 \\
Des. Est & \pm 2 & \pm 0.25 & \pm 0.26 & \pm 0.92 & \pm 0.89 \\
Mínimo & 6 & 0.68 & 0.77 & 2.0 & 2.7 \\
Máximo & 13 & 1.6 & 1.06 & 5.6 & 5.8 \\
\hline
\end{tabular}

Fuente: elaboración propia.

En la tabla 4 se muestran los resultados del componente cognitivo del test MoCA, con una normalidad de p > 0.05, el cual fue conglomerado en seis subgrupos, lo que permitió tener un resultado agrupado según cada ítem de denominación del test.

Tabla 4. Resultados descriptivos del componente cognitivo test MoCA

\begin{tabular}{lcccccc}
\hline & Visoespacial & $\begin{array}{c}\text { Denominación } \\
\text { animal }\end{array}$ & Memoria & Abstracción & Orientación & Lenguaje \\
\hline Media & 2.4 & 2.6 & 2.4 & 1.37 & 5.74 & 9.84 \\
Des. Est & \pm 1.1 & \pm 0.56 & \pm 1.80 & \pm 0.68 & \pm 0.52 & \pm 2.72 \\
Mínimo & 1 & 1 & 0 & 0 & 4 & 4 \\
Máximo & 3 & 3 & 5 & 2 & 6 & 14 \\
\hline
\end{tabular}

Fuente: elaboración propia.

Finalmente, en la tabla 5 se aprecian los coeficientes de correlación entre el componente morfológico y el componente cognitivo $(\mathrm{p}<0.05)$. Se evidencian niveles de correlación entre moderada y alta positiva $(\mathrm{r}=0.50 \mathrm{y}$ $r=0.85)$ y niveles de correlación alta negativa $(r=0.50$ y $r=0.85)$. 
Tabla 5. Coeficientes de correlación entre el componente morfológico y el test MoCA

\begin{tabular}{lccccccccc}
\hline Variables & & $\begin{array}{c}\text { Músculo } \\
\text { BI }\end{array}$ & $\begin{array}{c}\text { Músculo } \\
\text { PD }\end{array}$ & $\begin{array}{c}\text { Músculo } \\
\text { PI }\end{array}$ & Cintura & Talla & $\begin{array}{c}\text { Masa } \\
\text { magra } \\
(\mathbf{k g})\end{array}$ & $\begin{array}{c}\text { Masa } \\
\text { musculoes- } \\
\text { quelética } \\
(\mathbf{k g})\end{array}$ & $\begin{array}{c}\text { Grasa } \\
\text { visceral }\end{array}$ \\
\hline Visoespacial & r & & 0.725 & 0.695 & & 0.554 & 0.836 & $0.859^{*}$ & \\
& $\mathrm{p}$ & & 25 & 0.034 & & 0.034 & 0.023 & 0.046 & \\
Orientación & $\mathrm{r}$ & & 0.832 & 0.779 & -0.765 & & & & -0.809 \\
& $\mathrm{p}$ & & 0.026 & 0.045 & 0.037 & & & & 0.038 \\
Memoria & $\mathrm{r}$ & 0.819 & 0.853 & 0.806 & & 0.748 & 0.724 & $0.734 *$ & -0.819 \\
& $\mathrm{p}$ & 0.036 & 0.015 & 0.014 & & 0.017 & 0.032 & 0.025 & 0.018 \\
Recuerdo & $\mathrm{r}$ & 0.719 & 0.753 & 0.756 & & & 0.824 & 0.834 & \\
diferido & $\mathrm{p}$ & 0.046 & 0.045 & 0.034 & & & 0.024 & 0.025 & \\
& $\mathrm{r}$ & 0.819 & 0.853 & 0.856 & -0.865 & & 0.724 & 0.734 & \\
MoCA total & $\mathrm{p}$ & 0.026 & 0.035 & 0.034 & 0.034 & & 0.042 & 0.055 & \\
\hline
\end{tabular}

Fuente: elaboración propia.

\section{Rangos del componente morfológico}

Según los resultados, en el IMC se muestra una población en estado de sobrepeso, como se puede evidenciar en diferentes estudios exclusivos con población femenina y como se reporta en el trabajo de Guzmán-Saldaña, Saucedo-Molina, García, Galván y del Castillo (2017). El perímetro de la cintura muestra un grado de alto riesgo cardiometabólico, según las tablas de referencia estándar a nivel nacional, las cuales concuerdan con el estudio de Buendía y colaboradores (2016b). La masa grasa, reportada con el método de impedancia, evidencia rangos altos (Gómez-Ambrosi, et al., 2018), los cuales están relacionados con el riesgo metabólico y cardiovascular, como se ha manifestado en diferentes reportes académicos (Skrabal, et al., 2017). La masa muscular esquelética, como la masa muscular por segmentos, presenta niveles bajos para poder determinar una acción funcional normal o de independencia, como se muestra en el estudio de Bosy-Westphal, y 
colaboradores (2017), en el que se reporta un déficit de la masa muscular frente a la funcionalidad de los sujetos.

\section{Rangos del componente cognitivo}

La aplicación del test MoCA señaló un resultado promedio total por debajo de 26 puntos, lo cual reporta, en su tabla de baremación, un DCL moderadoalto. Esto reafirma el primer paso para la aparición de alzheimer (Holguin y Medina, 2017), como se evidencia en el estudio de validación de Delgado, Araneda, y Behrens (2017), quienes reportaron los niveles de evaluación y determinación del DCL en población adulta. Los hallazgos de la revisión sistemática de Loureiro y colaboradores (2018) concuerdan con los rangos de evaluación del DCL en población adulta.

La aplicación del test de MoCA indicó una correlación moderada a alta frente al componente morfológico, como se reporta en estudios de tipo hospitalario frente a la aparición de DCL y el componente morfológico (López, 2018). Otros estudios evidencian una asociación entre el DCL y el componente de masa muscular en unidades de cuidado hospitalario (Busico, et al., 2019), lo cual se asemeja con los resultados de correlación de este estudio.

\section{Conclusiones}

Se encontraron relaciones significativas en cuanto a la composición corporal y el DCL, principalmente frente al componente muscular y magro. En relación con el componente de la masa grasa se encuentra que, si existe un aumento en este tipo de tejido, el DCL se ve más afectado. Es claro que los malos resultados en el estado morfológico presentan repercusiones muy marcadas en el DCL, lo cual reafirma que es el primer paso para la aparición de alzheimer y, aún más, un déficit en la funcionalidad del adulto mayor, como se reporta en diferentes estudios. 


\section{Referencias}

Aristizábal, J. y Giraldo, A. (2017). Comparación de la composición corporal de mujeres jóvenes obtenida por hidrodensitometría y tres técnicas de bioimpedancia. Iatreia, 30(3), 255-64. DoI: https://doi.org/10.17533/udea.iatreia. v30n3a01

Bosy-Westphal, A., Jensen, B., Braun, W., Pourhassan, M., Gallagher, D. y Müller, M. (2017). Quantification of whole-body and segmental skeletal muscle mass using phase-sensitive 8-electrode medical bioelectrical impedance devices. European Journal of Clinical Nutrition, 71(9), 1061-67. DoI: https://doi. org/10.1038/ejcn.2017.27

Buendía, R., Zambrano, M., Díaz, Á., Reino, A., Ramírez, J. y Espinosa, E. (2016b). Waist circumference cut-off points for the diagnosis of abdominal obesity in Colombian population by means of bioimpedance as a reference standard. Revista Colombiana de Cardiología, 23(1), 19-25. DoI: https://doi. org/10.1016/j.rccar.2015.07.011

Busico, M., das Neves, A., Carini, F., Pedace, M., Villalba, D., Foster, C., García, J., Garbarini, M., Jereb, S., Sacha, V. y Estenssoro, E. (2019). Programa de seguimiento al alta de la unidad de cuidados intensivos. Medicina Intensiva, 43(4), 243-54. DoI: https://doi.org/10.1016/j.medin.2018.12.005

Delgado, C., Araneda, A. y Behrens, M. (2017). Validación del instrumento Montreal Cognitive Assessment en español en adultos mayores de 60 años. Neurología, 34(6). DoI: https://doi.org/10.1016/j.nrl.2017.01.013

Delgado, C., Araneda, A. y Behrens, M. I. (2019). Validation of the Spanishlanguage version of the Montreal Cognitive Assessment test in adults older than 60 years. Neurología (English Edition), 34(6), 376-85. Recuperado de https://doi.org/10.1016/j.nrleng.2018.12.008

Erickson, K., Hillman, C. y Kramer, A. (2015). Physical activity, brain, and cognition. Current Opinion in Behavioral Sciences, 4, 27-32. DoI: https://doi. org/10.1016/j.cobeha.2015.01.005

Freitas, S., Simões, M., Alves, L. y Santana, I. (2013). Montreal cognitive assessment: validation study for mild cognitive impairment and Alzheimer disease. Alzheimer Disease y Associated Disorders, 27(1), 37-43. DoI: $10.1097 /$ WAD.0b013e3182420bfe

Gaur, V. y Ranga, A. (2018). Statistically Significant of Diabetes with Body Mass Index. International Journal of Applied Engineering Research, 13(24), 16758-61. 
Gianoudis, J., Bailey, C. y Daly, R. (2015). Associations between sedentary behaviour and body composition, muscle function and sarcopenia in communitydwelling older adults. Osteoporosis International, 26(2), 571-9. DoI: https:// doi.org/10.1007/s00198-014-2895-y

Gómez-Ambrosi, J., González-Crespo, I., Catalán, V., Rodríguez, A., Moncada, R., Valentí, V., Romero, S., Ramírez, B., Silva, C., Gil, M., Salvador, J., Benito, A., Colina, I. y Frühbeck, G. (2018). Clinical usefulness of abdominal bioimpedance (ViScan) in the determination of visceral fat and its application in the diagnosis and management of obesity and its comorbidities. Clinical Nutrition, 37(2), 580-9. DoI: https://doi.org/10.1016/j.clnu.2017.01.010

Gómez, F., Zunzunegui, M., Lord, C., Alvarado, B. y García, A. (2013). Applicability of the MoCA-S test in populations with little education in Colombia. International Journal of Geriatric Psychiatry, 28(8), 813-20. DOI: https://doi. org/10.1002/gps.3885

Guzmán-Saldaña, R., Saucedo-Molina, T., García, M., Galván, M. y del Castillo, A. (2017). Imagen corporal e índice de masa corporal en mujeres indígenas del estado de Hidalgo, México. Revista Mexicana de Trastornos Alimentarios, 8(1), 56-62. DoI: http://dx.doi.org/10.1016/j.rmta.2017.01.002

Holguin, M. y Medina, S. (2017). Diagnóstico de deterioro cognitivo leve demencia Senil Alzheimer mediante la utilización de MMSE-MOCA (trabajo de grado). Universidad Estatal de Milagro. Milagro, Ecuador.

Hu, C., Yu, D., Sun, X., Zhang, M., Wang, L. y Qin, H. (2017). The prevalence and progression of mild cognitive impairment among clinic and community populations: a systematic review and meta-analysis. International Psychogeriatrics, 29(10), 1595-608. DoI: https://doi.org/10.1017/S1041610217000473

Huey, E., Manly, J., Tang, M., Schupf, N., Brickman, A., Manoochehri, M., Mex, J., DeCarli, C., Devanand, D. y Mayeux, R. (2013). Course and etiology of dysexecutive MCI in a community sample. Alzheimer's y Dementia, 9(6), 632-9. DOI: https://doi.org/10.1016/j.jalz.2012.10.014

Labraña, A., Durán, E., Martínez, M., Leiva, A., Garrido-Méndez, A., Díaz, X. y Celis-Morales, C. (2017). Menor peso corporal, de índice de masa corporal y de perímetro de cintura se asocian a una disminución en factores de riesgo cardiovascular en población chilena: Findings from the Chilean health surveyç. Revista Médica de Chile, 145(5), 585-94. DoI: http://dx.doi.org/10.4067/ S0034-98872017000500005

López, F, (2018). Nivel de reserva cognitiva y dependencia funcional en pacientes con accidente cerebro vascular isquémico de un hospital nacional (tesis inédita 
de especialización). Universidad Norbert Wiener. Lima, Perú. Recuperado de http://repositorio.uwiener.edu.pe/handle/123456789/2061

Loureiro, C., García, C., Adana, L., Yacelga, T., Rodríguez Lorenzana, A. y Maruta, C. (2018). Uso del test de evaluación cognitiva de Montreal (MoCA) en América Latina: revisión sistemática. Revista de Neurología, 66(12), 397408. DOI: https://doi.org/10.33588/rn.6612.2017508

Mitchell, A. y Shiri-Feshki, M. (2009). Rate of progression of mild cognitive impairment to dementia-meta-analysis of 41 robust inception cohort studies. Acta Psychiatrica Scandinavica, 119(4), 252-65. DoI: https://doi. org/10.1111/j.1600-0447.2008.01326.x

Montoya, S., González-Ballesteros, L., Flórez C., Galán, A., Sánchez, N., ValenciaLópez, N., Correa, L., Díaz, A., Alzate, P., Berrocal, L., Acevedo, I., Ruiz, C. y Botero, F. (2015). Misión Colombia envejece. Cifras retos y recomendaciones. Fundación Saldarriaga Concha. Bogotá, Colombia. Recuperado de https:// www.repository.fedesarrollo.org.co/bitstream/handle/11445/2724/LIB_2015 _MCE_completo.pdf? sequence $=5$ yisAllowed $=\mathrm{y}$

Parra, M., Baez, S., Allegri, R., Nitrini, R., Lopera, F., Slachevsky, A., Custodio, N., Lira, D., Kumfor, F., Huepe, D., Cogram, P., Bak, T., Manes, F. e Ibanez, A. (2018). Dementia in Latin America: Assessing the present and envisioning the future. Neurology, 90(5), 222-31. DoI: https://doi.org/10.1212/ WNL.0000000000004897

Pedraza, O., Salazar, A., Sierra, F., Soler, D., Castro, J., Castillo, P., Hernández, A. y Píñeros, C. (2016). Confiabilidad, validez de criterio y discriminante del Montreal Cognitive Assessment (MoCA) test, en un grupo de adultos de Bogotá. Acta Médica Colombiana, 41(4), 221-8. Recuperado de http://www. scielo.org.co/pdf/amc/v41n4/v41n4a04.pdf

Petersen, R., Lopez, O., Armstrong, M., Getchius, T., Ganguli, M., Gloss, D., Gronseth, G., Marson, D., Pringsheim, T., Day, G., Sager, M., Stevens, J. y RaeGrant, A. (2018). Practice guideline update summary: Mild cognitive impairment: Report of the Guideline Development, Dissemination, and Implementation Subcommittee of the American Academy of Neurology. Neurology, 90(3), 126-35. DoI: https://doi.org/10.1212/WNL.0000000000004826

Romo-Galindo, D. A. y Padilla-Moya, E. (2019). Use cognitive tests to detect people with dementia in mexican population. Archivos de Neurociencias, 23(4), 26-34. Recuperado de: https://www.medigraphic.com/cgi-bin/new/resumenI. cgi? IDARTICULO=86913 
Rodríguez-Romo, G., Barriopedro, M., Alonso, P. y Garrido-Muñoz, M. (2015). Relaciones entre Actividad Física y Salud Mental en la población adulta de Madrid. Revista de Psicología del Deporte, 24(2), 233-9. DoI: https://doi. org/10.5232/ricyde2010.02004

Sala, G., Inagaki, H., Ishioka, Y. L., Masui, Y., Nakagawa, T., Ikebe, K. y Gondo, Y. (2019). Psychometric Properties of the Montreal Cognitive Assessment (MoCA): A Comprehensive Investigation [Preprint]. Recuperado de https:// doi.org/10.31234/osf.io/7xyuv

Skrabal, F., Pichler, G. P., Penatzer, M., Steinbichl, J., Hanserl, A.-K., Leis, A. y Loibner, H. (2017). The Combyn ${ }^{\mathrm{TM}}$ ECG: Adding haemodynamic and fluid leads for the ECG. Part II: Prediction of total body water (TBW), extracellular fluid (ECF), ECF overload, fat mass (FM) and "dry" appendicular muscle mass (AppMM). Medical Engineering y Physics, 44, 44-52. Dor: https://doi. org/10.1016/j.medengphy.2017.03.002

Tartt, A., Fulmore, C., Liu, Y., Rosoklija, G., Dwork, A., Arango, V. René, H., Mann, J. y Boldrini, M. (2018). Considerations for assessing the extent of hippocampal neurogenesis in the adult and aging human brain. Cell Stem Cell, 23(6), 782-3. DoI: https://doi.org/10.1016/j.stem.2018.10.025

Zhou, S., Zhu, J., Zhang, N., Wang, B., Tao. L., Lv, X., Pin, T., Yu, X. y Wang, H. (2014). The influence of education on Chinese version of Montreal cognitive assessment in detecting amnesic mild cognitive impairment among older people in a Beijing rural community. The Scientific World Journal, 1-7. Dor: http:// dx.doi.org/10.1155/2014/689456 